\title{
The role of probiotics in digestive health
}

\author{
This article was published in the following Dove Press journal: \\ Nutrition and Dietary Supplements \\ 10 December 2015 \\ Number of times this article has been viewed
}

\section{Arthur C Ouwehand}

Active Nutrition, DuPont Nutrition and Health, Kantvik, Finland
Correspondence: Arthur C Ouwehand Active Nutrition, DuPont Nutrition and Health, Sokeritehtaantie 20, 02460 Kantvik, Finland

Tel +358 405956353

Email arthur.ouwehand@dupont.com
Abstract: Although probiotics have documented health benefits outside the gastrointestinal tract, digestive health remains the key benefit for probiotics. Advances in technology have made even more in-depth analyses of the intestinal microbiota possible. Nevertheless, a healthy microbiota cannot be defined due to individual differences and changes with age. Probiotics, in general, will not cure or prevent disease, but will aim at maintaining health and reducing risk for disease; although probiotic pharmaceuticals are possible. Meta-analyses have indicated several areas where probiotics can exert health benefits such as certain types of diarrhea, constipation, and inflammatory diseases of the intestine. There is also an ever-increasing understanding of the mechanisms of probiotic action. However, it remains challenging to pinpoint specific strain (combinations) for explicit health benefits. The increased understanding of the intestinal microbiota composition and activity will make it likely that in the future we will see new probiotics from genera other than Lactobacillus, Bifidobacterium, or Saccharomyces; these may focus on new or existing health targets.

Keywords: Lactobacillus, Bifidobacterium, intestinal microbiota, health, novel probiotics

\section{Introduction}

The most widely accepted definition for probiotics is the one proposed by the Food and Agriculture Organization/World Health Organization working group and recently reaffirmed by the International Scientific Association for Probiotics and Prebiotics: live microorganisms that, when administered in adequate amounts, confer a health benefit on the host. ${ }^{1,2}$ The definition thus highlights three major points: 1) the viability of the microbes, 2) the dose, and 3) the documented health benefit.

\section{Viability}

The viability of the microbes sets certain technological challenges. Most probiotics belong to the genera Lactobacillus and Bifidobacterium, although strains and species from other genera are also being used. This requires that the microbes remain viable until the end of shelf life. Probiotics are commonly consumed in two main formats: as a food and as a dietary supplement. Foods, in general, have a high water activity and the fermented foods have a low $\mathrm{pH}$. Such foods will often require refrigerated storage and have a limited shelf life; weeks rather than months or years. In the case of dietary supplements, the water activity is usually low, but the products tend to be stored at ambient temperatures and may have shelf lives of up to 2 years. Until the end of shelf life, a sufficient dose needs to be available. Preferably, this is mentioned on the product; the amount of viable microbes at time of manufacture is of less interest 
as it does not indicate what the minimal dose is at time of consumption.

\section{Dose}

Dose is thus important. However, what this dose should be is not always that clear. A rule of thumb is that the dose should be at least $10^{9}$ colony-forming units, although Health Canada recommends at least $10^{7}$ colony-forming units for a general probiotic claim. ${ }^{3}$ However, the dose is likely to be dependent on the particular probiotic strain (combination), the health benefit, and subpopulation the consumer belongs to. Therefore, the dose that has been documented to provide a health benefit should be taken as per the guidelines; as is also required by Health Canada for specific health claims.

\section{Health benefit}

Finally, there is the health benefit; what constitutes a health benefit and what not? At the time of writing (October 2015), the European Food Safety Authority did not approve health claims for probiotics. This should, however, not be interpreted in a way that probiotics do not provide health benefits. An in-depth analysis of the regulatory challenges of probiotic health claims in various jurisdictions falls outside the scope of this work. For simplicity, here, a health benefit will be interpreted as an improvement in a clinical outcome. This means that changes in the microbiota composition or certain immune markers will not be considered. The strength of such markers is not generally accepted. ${ }^{4}$

\section{Safety}

Although not mentioned in the definition, it is obvious that probiotics are commercialized should be well identified and safe. Identification should be done by molecular techniques. Although not required, genome sequencing is nowadays so cost-effective that it would not be excessive to request for commercial probiotic strains. For members of the genera Bifidobacterium and Lactobacillus, safety is not an issue for the general population. For consumers (patients) with a poor general health and in particular a poor immune status, a riskbenefit consideration should be made. This population tends to have the greatest potential benefit from probiotics, and risk (usually infection) remains small. In the unlikely event an infection should happen, it can be easily treated. Finally, for the benefit of all consumers, commercialized probiotics should not harbor transferable antibiotic resistance. This is something that can be tested both phenotypically and through genome analysis.

\section{Strain specificity versus commonality}

Most commercial probiotics belonging to the narrow group of lactic acid bacteria, lactobacilli and bifidobacteria, have been isolated from similar sources like fermented dairy products or intestine, and have generally been selected to survive low $\mathrm{pH}$ and bile acids. It is therefore reasonable to assume that different strains share some properties, ${ }^{5}$ while other characteristics may indeed be specific for a particular strain; this is schematically represented in Figure 1.

\section{Intestinal microbiota}

Techniques to analyze the intestinal microbiota composition and activity are continuously improving; enabling analyses that were until recently considered impossible. An in-depth review of the intestinal microbial analyses falls outside the scope of this work and the reader is referred to other sources for this, for example, Lankelma et al. ${ }^{6}$ However, there is general agreement that the intestinal microbiota, which by the way is the correct term for what used to be referred to as the intestinal microflora (we are dealing with small life, not small plants), plays a major role in health and disease and in much more areas than previously thought. Intestinal microbiota often refers to fecal microbiota, therefore, it is important to realize that different parts of the gastrointestinal tract harbor different microbiota.

Despite developments in analytical techniques, it has been difficult to pinpoint specific organisms or groups of organisms that are causally associated with certain health conditions. This is one of the reasons why changing the composition of the microbiota is often not considered a health benefit as it is not clear what change is desired; not even increasing levels of bifidobacteria and/or lactobacilli. One thing, however, becomes clear; in general, diversity is good. Many disease conditions appear to be associated with a

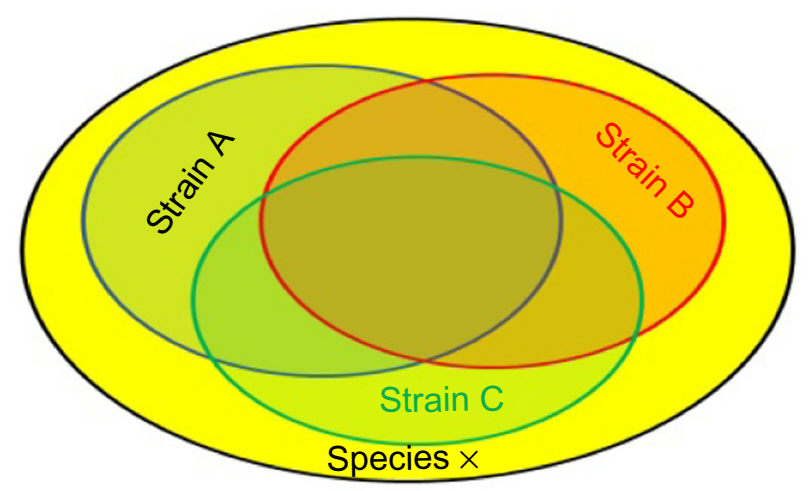

Figure I Schematic representation of common properties of different strains within a given species.

Notes: A similar analogy can be used for common properties between strains from different species within the same genus or taxonomic group. 
reduced microbial diversity. ${ }^{7}$ This is not a surprise; in ecology, diversity in an ecosystem is associated with resilience and monocultures are susceptible to disturbances.

Although there is a general agreement that the intestinal microbiota plays a prominent role in health and disease, the exact mechanisms and contributions by the various members are still only poorly understood. This is also complicated by the fact that every individual has his or her own personal microbiota; there is no "standard" healthy microbiota as it would be different for everyone and changes during our life. ${ }^{8}$

Some probiotics have been shown to change the composition of the intestinal microbiota. This does, however, not always happen. ${ }^{9}$ A reason for this could be that a healthy microbiota, whatever it is, is able to resist changes induced by outside factors; both good (probiotics) and bad (pathogens). When a microbiota is disturbed, probiotics may help in its recovery, that is, returning to its original state.$^{10} \mathrm{~A}$ clear example of this is in association with antibiotic use (see Antibiotic-associated diarrhea section). While most commercial probiotics have been documented to survive gastrointestinal transit, this in itself is not a health benefit and also does not explain how they can contribute to microbiota recovery as probiotics are not simply supplementing depleted species. Rather probiotics appear to be contributing to an improvement in the local environment that allows the microbiota to recover.

\section{Digestive health}

Digestive health is not formally defined. In practice, it should be defined through the absence of intestinal complaints. This is clearly not satisfying, but may work from a practical perspective. Most healthy consumers will be happy with their digestive health if they are not having any symptoms, that is, are not reminded of their intestines in the first place.

Therefore, digestive health therefore focuses on reducing risk for gastrointestinal disturbances and/or relieving such symptoms if and when they occur. This is discussed in the next section.

Although probiotics can be used as a drug, most probiotics are marketed as food ingredients or as dietary supplements. ${ }^{1}$ As such, probiotics are not expected to prevent disease, they may reduce the risk of disease. Probiotics do not treat or mitigate disease, they may relieve symptoms of disease. Therefore, most probiotics are not treated as drug, their main aim is to maintain health. Furthermore, traditional fermented foods as such yogurt, sauerkraut, and kimchi are not probiotics, unless they contain specific strains with documented health benefits such as certain probiotic yogurts (Figure 2).

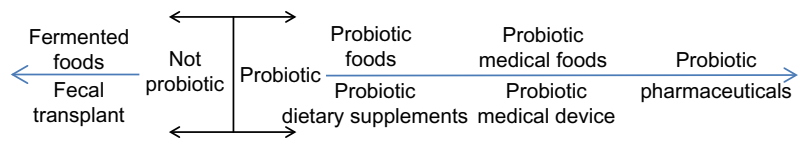

Figure 2 Schematic representation of probiotic products; distinguishing nonprobiotic products with live microbes from probiotic products and indicating the range from food/dietary supplement to pharma.

\section{Constipation, slow intestinal transit}

Constipation is one of the most common digestive disorders. Although benign, constipation has a substantial influence on general well-being and is considered as a risk for certain serious intestinal conditions such as diverticulitis. Constipation is defined as three or less spontaneous bowel movements per week. It is more common in females than in males and tends to increase with age. Even though clearly defined, constipation is to some extent subjective and difficult to measure. Alternative measures are the Bristol Stool Scale ${ }^{11}$ and intestinal transit time. Intestinal transit time can be measured with various techniques, using dyes such as carmine red, radiopaque markers, and so-called smart pills. The former determines oroanal transit time and is not very sensitive as subjects tend to defecate once a day or less. ${ }^{12}$ Radiopaque markers are sensitive, but require that subjects are compliant with their consumption of the markers for 6 days (to create a steady state) and involves exposure to radiation..$^{13}$ The socalled smart pill provides more information than just colonic transit time; ${ }^{14}$ the technique is, however, expensive and has not been routinely used for measuring transit for research purposes. Most probiotic studies on intestinal transit have therefore used radiopaque markers and abdominal X-ray.

A number of probiotics have been tested for their effect on slow intestinal transit; most of them are Bifidobacterium lactis strains, such as B. lactis DN-173010, B. lactis HN019, or the combination of Bifidobacterium longum BB536 and B. lactis $420 .{ }^{15}$ In general, the tested probiotics have been found to shorten colonic transit. ${ }^{16,17}$ Important here is that the probiotics do not shorten the transit time of subjects with "normal" initial transit times, that is, they do not cause diarrhea (Figure 3). Probiotics have also been shown to improve stool frequency and consistency. ${ }^{18}$ Although studies have used many different doses, meta-analyses have failed to observe a dose-response effect. ${ }^{17}$

\section{Antibiotic-associated diarrhea}

Antibiotic-associated diarrhea (AAD) has been reported to occur between $5 \%$ and $39 \%$ of patients on antibiotics. ${ }^{19}$ However, the source data for these numbers is relatively old and focuses on hospitalized patients. A more recent 


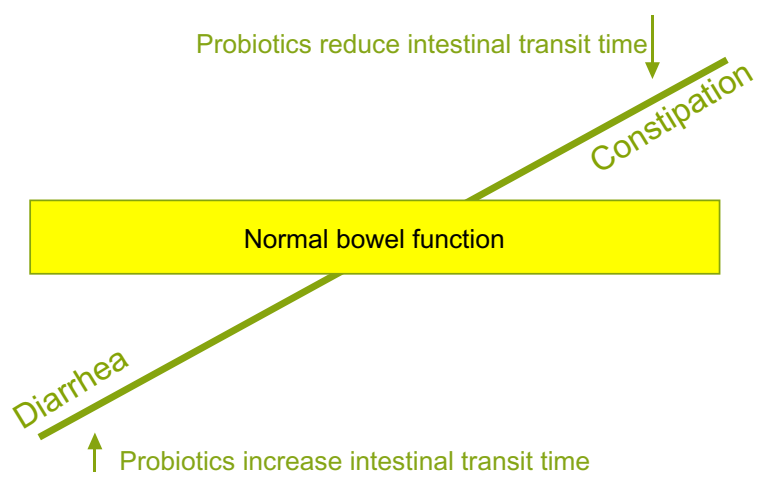

Figure 3 Principle by which probiotics can both provide benefits for diarrhea and constipation; normalization of the intestinal transit.

epidemiological investigation found that $9.6 \%$ of hospital patients that were prescribed antibiotics developed AAD; mainly patients 65 years and older. The AAD incidence in ambulatory patients is less known. Nevertheless, AAD affects quality of life and causes a substantial cost to the health care system. Thus, complementary therapies that can reduce its incidence are certainly welcome.

The most common pathogens causing $\mathrm{AAD}$ are assumed to be Clostridium difficile, Clostridium perfringens, and Staphylococcus aureus. However, these pathogens do not account for all AAD and thus many unknown causes remain; some of which may be nonmicrobiological and relate to physiological changes in the host (eg, changed bile acid resorption).

Probiotics have been shown to be very successful in reducing risk for $\mathrm{AAD} .{ }^{20}$ When looking at the settings, most studies have been performed in a clinical/hospital setting and have proved to be successful. Much less data are available on community settings and ambulatory patients. ${ }^{21}$ The use of probiotics has been calculated to reduce cost for diarrhea treatment by $25 \%,{ }^{22}$ this is associated with a number needed to treat of 13 .

In general, probiotic combinations, such as Bifidobacterium bifidum $\mathrm{W} 23+$ B. lactis $\mathrm{W} 18+$ B. longum $\mathrm{W} 51+$ Enterococcus faecium $\mathrm{W} 54+$ Lactobacillus acidophilus $\mathrm{W} 37+$ L. acidophilus $\mathrm{W} 55$ + Lactobacillus paracasei $\mathrm{W} 72$ + Lactobacillus plantarum $\mathrm{W} 62+$ Lactobacillus rhamnosus $\mathrm{W} 71+$ Lactobacillus salivarius $\mathrm{W} 24^{23}$ or L. acidophilus $\mathrm{NCFM}+$ L. paracasei Lpc-37+ B. lactis $\mathrm{Bi}-07+$ B. lactis $\mathrm{BL}-04^{24}$ or L. acidophilus CL1285 and Lactobacillus casei LBC80 ${ }^{25}$ have been shown to be successful. Among the single strains, L. rhamnosus GG has been shown to help; ${ }^{26}$ E. faecium SF68 has shown varying results. ${ }^{27}$ Also yeasts, in particular Saccharomyces boulardii (nom. inval.), have been reported to be efficacious in reducing AAD risk. ${ }^{20}$ The challenge here is that until recently no strain designations were reported in S. boulardii.
Many more probiotic strains and combinations have been shown to be efficacious and it is beyond the scope of this review to describe them all. For further details the reader is referred to the study by Hempel et al. ${ }^{20}$

Interestingly, in $\mathrm{AAD}$, two rare probiotic dose-response studies have been performed..$^{24,25}$ Both studies find that the higher dose was more efficacious than the lower tested dose. Although this should not be generalized, it is interesting to note that dose-response effects have been observed with probiotics.

\section{Community-acquired diarrhea}

Community-acquired diarrhea can be caused by a multitude of pathogens, toxins, and other factors. Some of the best investigated community-acquired diarrhea targets for probiotics are rotavirus diarrhea and traveler's diarrhea.

L. rhamnosus $\mathrm{GG}$ is an example of a probiotic that has been shown to reduce the risk for rotavirus diarrhea in children in a hospital setting (ie, a nosocomial infection) ${ }^{28}$ and has also been observed to aid in the treatment of rotavirus diarrhea. ${ }^{29}$ For reducing the risk of rotavirus infection in day care centers, the evidence is minimal to modest ${ }^{30}$ for all strains tested. With the availability of a rotavirus vaccine, it is uncertain to what extent this is still a relevant target, both in hospital and community settings. In community-acquired diarrhea, the etiology is often not determined, but also with these diarrheas, probiotics such as B. lactis $\mathrm{HN} 019^{31,32}$ or L. paracasei Lpc-3731 have been observed to be successful in reducing risk, especially in young children.

Traveler's diarrhea has a plethora of causes; it is therefore not surprising that probiotics are less successful in preventing this type of diarrhea, with an estimated (nonsignificant) reduction of $8 \% .{ }^{21}$ In addition to this, the studies that have been used in evaluating the efficacy suffer from high attrition rates.

However, where efficacious, the use of probiotics has been calculated to reduce cost for diarrhea treatment by $25 \%$. While initial cost was increased (cost for the probiotic), subsequent additional medication and consultations were reduced. ${ }^{22}$

It may seem contradictory that a probiotic can both reduce transit time in the case of constipation and prolong it in the case of diarrhea. However, a more correct perspective is that probiotics normalize the transit time (Figure 3). ${ }^{33}$

\section{Irritable bowel syndrome}

Irritable bowel syndrome (IBS), not to be confused with inflammatory bowel disease (IBD), is not a disease but a syndrome. IBS is defined according to the Rome III 
criteria as: recurrent abdominal pain or discomfort at least 3 days per month in the last 3 months associated with two or more of the following:

- Improvement with defecation

- Onset associated with a change in frequency of stool

- Onset associated with a change in form (appearance) of stool.

Furthermore, there is no evidence of an inflammatory, anatomic, metabolic, or neoplastic process that explains the subject's symptoms. IBS can be subtyped into diarrheal, constipation, or mixed type using the Bristol Stool Scale ${ }^{34}$ or may be undefined. As there is no clear etiology, treatment is challenging, even though IBS-like symptoms are among the most common reasons for visiting gastroenterologists. IBS appears to have a strong psychosomatic component and is therefore prone to a placebo effect; this necessitates that studies are sufficiently long (several months) and have sufficient numbers of volunteers (hundreds of people/treatment group). It may also be necessary to focus on one IBS subtype in order to have a sufficiently homogenous study population. Probiotics appear to have potential in relieving specific symptoms of IBS, such as abdominal pain (eg, VSL\#3 $3^{35}$ or Bacillus coagulans GBI-30, 6086), ${ }^{36}$ bloating (eg, L. acidophilus $\mathrm{NCFM}+$ B. lactis $\mathrm{Bi}-07^{37}$ or B. bifidum MIMBb75), ${ }^{38}$ and flatulence (eg, $S$. boulardii). ${ }^{39}$ Overall improvement in IBS quality of life, however, appears to be challenging. ${ }^{40,41}$ It is therefore also difficult to identify specific strains or strain combinations that would be most efficacious. However, as indicated earlier, selected strains have been documented to provide relief for selected symptoms of IBS.

\section{Necrotizing enterocolitis}

Necrotizing enterocolitis (NEC) is a very serious condition with a high mortality rate. The main risk group is very low birth weight infants $(<1500 \mathrm{~g})$. Fortunately, NEC is rare among live births in general $(<0.1 \%)$. The pathogenesis of NEC is not fully understood, but likely to be multifactorial with an important microbial component. The intestinal microbiota of preterm infants has been reported to be less diverse than that of term infants and appears to be more commonly colonized by potential pathogenic species from genera such as Klebsiella, Enterobacter, and Clostridium..$^{42}$ There is currently no treatment for the prevention of NEC. However, probiotics have been reported to be very successful in reducing NEC risk in the at-risk population and is actually one of the best documented benefits of probiotics. ${ }^{43}$ Because of the involvement of potential pathogens in the etiology of NEC, administration of probiotics should start as early as possible and continue until at least the corrected gestational age of 36 or 37 weeks. ${ }^{44}$ Since most studies are relatively small and doses and study designs are varying, it is difficult to recommend specific strains for this application, on the other hand, it appears that most tested strain have been successful. This has led to the ever more widely accepted opinion that probiotics should be used routinely in the risk reduction of NEC. ${ }^{45}$ Despite this, the population is a very sensitive one that may suggest the need for more stringent quality control of the probiotic product; what this control should encompass in terms of microbiological safety testing remains to be determined. Furthermore, adverse events should be documented.

\section{Inflammatory bowel disease}

IBD can be divided into three different conditions: ulcerative colitis, which is an inflammatory condition limited to the colon; Crohn's disease (CD), which is an inflammation that can be anywhere in the intestinal tract; and pouchitis, which is an inflammation of an ileoanal pouch.

IBD is characterized by periods of active disease, which is treated, and periods of remission. The etiology of IBD is not known, but the microbiota appears to play an important role. Hence, it is not surprising that probiotics have been considered for IBD. The main probiotics studied are Escherichia coli Nissle 1917 and the probiotic combination VSL\#3. The former appears to be efficacious in maintaining remission in $\mathrm{CD}$, while the latter appears to be efficacious in both inducing and maintaining remission in $\mathrm{CD} ;{ }^{46}$ for inducing remission in combination with conventional therapy and maintaining remission in ulcerative colitis; ${ }^{47,48}$ and maintaining remission in pouchitis. ${ }^{47}$

\section{Helicobacter pylori eradication}

Helicobacter pylori is a common inhabitant of the gastric mucosa and has been found to be associated with gastric ulcer and gastric cancers. However, H. pylori has a dual role: exposure to the organism has been found to correlate with reduced risk for atopic dermatitis in children ${ }^{49}$ and eradication of $H$. pylori has been suggested to correlate with an increased risk of obesity ${ }^{50}$ It is because of its pathogenic character that it is eradicated from carriers. While in vitro and animal studies have indicated antagonistic activities toward $H$. pylori by, in particular, Lactobacillus probiotics, this has not been replicated in humans. Probiotics can therefore not be considered as an alternative for standard therapy. ${ }^{51}$ Some strains such as Lactobacillus johnsonii $\mathrm{La}^{52}$ have been observed to reduce $H$. pylori numbers and activity, which may actually be preferable in light of the potential positive role the organism may play. 
While not eradicating, probiotics have been found to be promising as adjuvants to standard therapy, reducing side effects, thereby improving compliance and leading to a higher success rate $(72 \%$ vs $82 \%) .{ }^{53}$ This is also suggested in the Maastricht IV consensus report on treatment of $H$. pylori. ${ }^{54}$ The Maastricht IV report recommends (with recommendation grade D) the use of $S$. boulardii. The positive effect of certain probiotics in the eradication of $H$. pylori can be explained by similar mechanisms as for AAD risk reduction, though other factors seem to be involved as well.

\section{Future}

The continued in-depth analysis of the intestinal microbiota is likely to indicate new probiotic candidates. Comparison of microbiota between different populations not only indicates which organisms are positively associated with disease, but also which organisms are missing or reduced in numbers. Such organisms may have probiotic potential. Examples of this include Akkermansia muciniphila, a mucus degrading organism with anti-inflammatory potential that may play a role in reducing metabolic syndrome. Another example is Faecalibacterium prausnitzii, which also appears to have anti-inflammatory potential and maybe useful in CD.

Genetically modified probiotics have been tested already some time ago, such as IL-10 producing L. lactis strains and while successful ${ }^{55}$ this does not appear to have led to any greater success. Obviously, such probiotics are in the pharmaceutical range of the spectrum (Figure 2).

\section{Conclusion}

Digestive health is the "traditional" target for probiotics. Some health benefits seem to be fairly well established for a variety of stains, especially in infants and children, such as constipation/long transit, AAD, certain forms of communityacquired diarrhea, certain IBS symptoms, and NEC. With the exception of VSL\#3 for IBD, it is not always easy to identify which strain (combination) is most efficacious. For the consumer, it therefore remains challenging to identify the appropriate product; some things to look for are numbers guaranteed until end of shelf life, and are strains indicated or just species and/or genus?

\section{Disclosure}

The author is an employee of DuPont Nutrition and Health. DuPont Nutrition and Health manufactures and commercializes probiotics. The author reports no other conflicts of interest in this work.

\section{References}

1. Hill C, Guarner F, Reid G, et al. Expert consensus document: The International Scientific Association for Probiotics and Prebiotics consensus statement on the scope and appropriate use of the term probiotic. Nat Rev Gastroenterol Hepatol. 2014;11(8):506-514.

2. FAO/WHO, editor. Guidelines for the Evaluation of Probiotics in Food; 2002. Available from: http://www.who.int/foodsafety/fs_management/ en/probiotic_guidelines.pdf. Accessed November 25, 2015.

3. Natural Health Products; Probiotics monograph, (2015).

4. Albers R, Bourdet-Sicard R, Braun D, et al. Monitoring immune modulation by nutrition in the general population: identifying and substantiating effects on human health. Br J Nutr. 2013;110(Suppl 2): S1-S30.

5. Glanville J, King S, Guarner F, Hill C, Sanders ME. A review of the systematic review process and its applicability for use in evaluating evidence for health claims on probiotic foods in the European Union. Nutr J. 2015;14:16.

6. Lankelma JM, Nieuwdorp M, de Vos WM, Wiersinga WJ. The gut microbiota in internal medicine: implications for health and disease. Neth J Med. 2015;73(2):61-68.

7. Sankar SA, Lagier JC, Pontarotti P, Raoult D, Fournier PE. The human gut microbiome, a taxonomic conundrum. Syst Appl Microbiol. 2015;38(4):276-286

8. Ottman N, Smidt H, de Vos WM, Belzer C. The function of our microbiota: who is out there and what do they do? Front Cell Infect Microbiol. 2012;2:104.

9. Derrien M, van Hylckama Vlieg JE. Fate, activity, and impact of ingested bacteria within the human gut microbiota. Trends Microbiol. 2015;23(6):354-366

10. Engelbrektson A, Korzenik JR, Pittler A, et al. Probiotics to minimize the disruption of faecal microbiota in healthy subjects undergoing antibiotic therapy. J Med Microbiol. 2009;58(Pt 5):663-670.

11. Lewis SJ, Heaton KW. Stool form scale as a useful guide to intestinal transit time. Scand J Gastroenterol. 1997;32(9):920-924.

12. Hengst C, Ptok S, Roessler A, Fechner A, Jahreis G. Effects of polydextrose supplementation on different faecal parameters in healthy volunteers. Int J Food Sci Nutr. 2009;60(Suppl 5):96-105.

13. Cremonini F, Mullan BP, Camilleri M, Burton DD, Rank MR. Performance characteristics of scintigraphic transit measurements for studies of experimental therapies. Aliment Pharmacol Ther. 2002; 16(10):1781-1790

14. Rao SS, Kuo B, McCallum RW, et al. Investigation of colonic and whole-gut transit with wireless motility capsule and radiopaque markers in constipation. Clin Gastroenterol Hepatol. 2009;7(5):537-544.

15. Waller PA, Gopal PK, Leyer GJ, et al. Dose-response effect of Bifidobacterium lactis HN019 on whole gut transit time and functional gastrointestinal symptoms in adults. Scand J Gastroenterol. 2011;46(9):1057-1064.

16. Ouwehand AC, Forssten S, Nexmann Larsen C, Philipp S. Probiotics and its effect on slow colonic transit. J Food Nutr Disord. 2014;S1:1.

17. Miller LE, Ouwehand AC. Probiotic supplementation decreases intestinal transit time: meta-analysis of randomized controlled trials. World J Gastroenterol. 2013;19(29):4718-4725.

18. Dimidi E, Christodoulides S, Fragkos KC, Scott SM, Whelan K. The effect of probiotics on functional constipation in adults: a systematic review and meta-analysis of randomized controlled trials. Am J Clin Nutr. 2014;100(4):1075-1084.

19. McFarland LV. Evidence-based review of probiotics for antibioticassociated diarrhea and Clostridium difficile infections. Anaerobe. 2009;15(6):274-280.

20. Hempel S, Newberry SJ, Maher AR, et al. Probiotics for the prevention and treatment of antibiotic-associated diarrhea: a systematic review and meta-analysis. JAMA. 2012;307(18):1959-1969.

21. Sazawal S, Hiremath G, Dhingra U, Malik P, Deb S, Black RE. Efficacy of probiotics in prevention of acute diarrhoea: a meta-analysis of masked, randomised, placebo-controlled trials. Lancet Infect Dis. 2006;6(6):374-382. 
22. Vandenplas Y, De Hert S; Probiotical Study Group. Cost/benefit of synbiotics in acute infectious gastroenteritis: spend to save. Benef Microbes. 2012;3(3):189-194.

23. Koning CJ, Jonkers DM, Stobberingh EE, Mulder L, Rombouts FM, Stockbrugger RW. The effect of a multispecies probiotic on the intestinal microbiota and bowel movements in healthy volunteers taking the antibiotic amoxycillin. Am J Gastroenterol. 2008;103(1):178-189.

24. Ouwehand AC, DongLian C, Weijian X, et al. Probiotics reduce symptoms of antibiotic use in a hospital setting: a randomized dose response study. Vaccine. 2014;32(4):458-463.

25. Gao XW, Mubasher M, Fang CY, Reifer C, Miller LE. Dose-response efficacy of a proprietary probiotic formula of Lactobacillus acidophilus CL1285 and Lactobacillus casei LBC80R for antibiotic-associated diarrhea and Clostridium difficile-associated diarrhea prophylaxis in adult patients. Am J Gastroenterol. 2010;105(7):1636-1641.

26. Vanderhoof JA, Whitney DB, Lantonson DL, Hanner TL, Lupo JV, Young RJ. Lactobacillus GG in the prevention of antibiotic associated diarrhoea in children. J Pediatr. 1999;135(5):564-568.

27. Wunderlich PF, Braun L, Fumagalli I, et al. Double-blind report on the efficacy of lactic acid-producing Enterococcus SF68 in the prevention of antibiotic-associated diarrhoea and in the treatment of acute diarrhoea. J Int Med Res. 1989;17(4):333-338.

28. Szajewska H, Wanke M, Patro B. Meta-analysis: the effects of Lactobacillus rhamnosus GG supplementation for the prevention of healthcare-associated diarrhoea in children. Aliment Pharmacol Ther. 2011;34(9):1079-1087.

29. Isolauri E, Juntunen M, Rautanen T, Sillanaukee P, Koivula T. A human Lactobacillus strain ( Lactobacillus casei sp strain GG) promotes recovery from acute diarrhea in children. Pediatrics. 1991;88(1):90-97.

30. Guandalini S. Probiotics for prevention and treatment of diarrhea. J Clin Gastroenterol. 2011;45(Suppl):S149-S153.

31. Hemalatha R, Ouwehand AC, Forssten SD, et al. A community-based randomized double blind controlled trial of Lactobacillus paracasei and Bifidobacterium lactis on reducing risk for diarrhea and fever in preschool children in an Urban Slum in India. Eur J Nutr Food Safety. 2014;4:325-341

32. Sazawal S, Dhingra U, Hiremath G, et al. Prebiotic and probiotic fortified milk in prevention of morbidities among children: communitybased, randomized, double-blind, controlled trial. PloS One. 2010;5(8): e12164.

33. Ouwehand AC. Probiotics for intestinal and respiratory health. NutraCos. 2014(July/August):2-4.

34. Drossman DA. Rome III: the new criteria. Chin J Dig Dis. 2006;7(4): $181-185$

35. Michail S, Kenche H. Gut microbiota is not modified by randomized, double-blind, placebo-controlled trial of VSL\#3 in diarrheapredominant irritable bowel syndrome. Probiotics Antimicrob Proteins. 2011;3(1):1-7.

36. Hun L. Bacillus coagulans significantly improved abdominal pain and bloating in patients with IBS. Postgrad Med. 2009;121(2):119-124.

37. Ringel-Kulka T, Palsson OS, Maier D, et al. Probiotic bacteria Lactobacillus acidophilus NCFM and Bifidobacterium lactis Bi-07 versus placebo for the symptoms of bloating in patients with functional bowel disorders: a double-blind study. J Clin Gastroenterol. 2011;45(6):518-525.
38. Guglielmetti S, Mora D, Gschwender M, Popp K. Randomised clinical trial: Bifidobacterium bifidum MIMBb75 significantly alleviates irritable bowel syndrome and improves quality of life - a double-blind, placebocontrolled study. Aliment Pharmacol Ther. 2011;33(10):1123-1132.

39. Choi CH, Jo SY, Park HJ, Chang SK, Byeon JS, Myung SJ. A randomized, double-blind, placebo-controlled multicenter trial of Saccharomyces boulardii in irritable bowel syndrome: effect on quality of life. J Clin Gastroenterol. 2011;45(8):679-683.

40. Ortiz-Lucas M, Tobias A, Saz P, Sebastian JJ. Effect of probiotic species on irritable bowel syndrome symptoms: a bring up to date meta-analysis. Rev Esp Enferm Dig. 2013;105(1):19-36.

41. Ford AC, Quigley EM, Lacy BE, et al. Efficacy of prebiotics, probiotics, and synbiotics in irritable bowel syndrome and chronic idiopathic constipation: systematic review and meta-analysis. Am J Gastroenterol. 2014;109(10):1547-1561; quiz 1546, 1562.

42. Ouwehand AC. What role for probiotics in necrotising enterocolitis. Arch Pediatr Infect Dis. 2014;2(1):e14912.

43. Shane AL, Deshpande GC, Merenstein D. Improved neonatal outcomes with probiotics. JAMA Pediatr. 2013;167(10):885-886.

44. Deshpande GC, Rao SC, Keil AD, Patole SK. Evidence-based guidelines for use of probiotics in preterm neonates. BMC Med. 2011;9:92.

45. Robinson J. Cochrane in context: probiotics for prevention of necrotizing enterocolitis in preterm infants. Evid Based Child Health. 2014; $9(3): 672-674$.

46. Jonkers D, Penders J, Masclee A, Pierik M. Probiotics in the management of inflammatory bowel disease: a systematic review of intervention studies in adult patients. Drugs. 2012;72(6):803-823.

47. Shen J, Zuo ZX, Mao AP. Effect of probiotics on inducing remission and maintaining therapy in ulcerative colitis, Crohn's disease, and pouchitis: meta-analysis of randomized controlled trials. Inflamm Bowel Dis. 2014;20(1):21-35.

48. Guandalini S, Cernat E, Moscoso D. Prebiotics and probiotics in irritable bowel syndrome and inflammatory bowel disease in children. Benef Microbes. 2015;6(2):209-217.

49. Seiskari T, Kondrashova A, Viskari H, et al; EPIVIR Study Group. Allergic sensitization and microbial load - a comparison between Finland and Russian Karelia. Clin Exp Immunol. 2007;148(1):47-52.

50. Nwokolo CU, Freshwater DA, O’Hare P, Randeva HS. Plasma ghrelin following cure of Helicobacter pylori. Gut. 2003;52(5):637-640.

51. Franceschi F, Cazzato A, Nista EC, et al. Role of probiotics in patients with Helicobacter pylori infection. Helicobacter. 2007;12(Suppl 2): 59-63.

52. Cruchet S, Obregon MC, Salazar G, Diaz E, Gotteland M. Effect of the ingestion of a dietary product containing Lactobacillus johnsonii La1 on Helicobacter pylori colonization in children. Nutrition. 2003; 19(9):716-721.

53. Zhang MM, Qian W, Qin YY, He J, Zhou YH. Probiotics in Helicobacter pylori eradication therapy: a systematic review and meta-analysis. World J Gastroenterol. 2015;21(14):4345-4357.

54. Malfertheiner P, Megraud F, O'Morain CA, et al; European Helicobacter Study Group. Management of Helicobacter pylori infection - the Maastricht IV/Florence consensus report. Gut. 2012;61(5):646-664.

55. Braat H, Rottiers P, Hommes DW, et al. A phase I trial with transgenic bacteria expressing interleukin-10 in Crohn's disease. Clin Gastroenterol Hepatol. 2006;4(6):754-759.
Nutrition and Dietary Supplements

\section{Publish your work in this journal}

Nutrition and Dietary Supplements is an international, peer-reviewed, open access journal focusing on research into nutritional requirements in health and disease, impact on metabolism and the identification and optimal use of dietary strategies and supplements necessary for normal growth and development. The journal welcomes papers covering

\section{Dovepress}

original research, basic science, clinical \& epidemiological studies, reviews and evaluations, guidelines, expert opinion and commentary, case reports and extended reports. The manuscript management system is completely online and includes a very quick and fair peer-review system, which is all easy to use. 